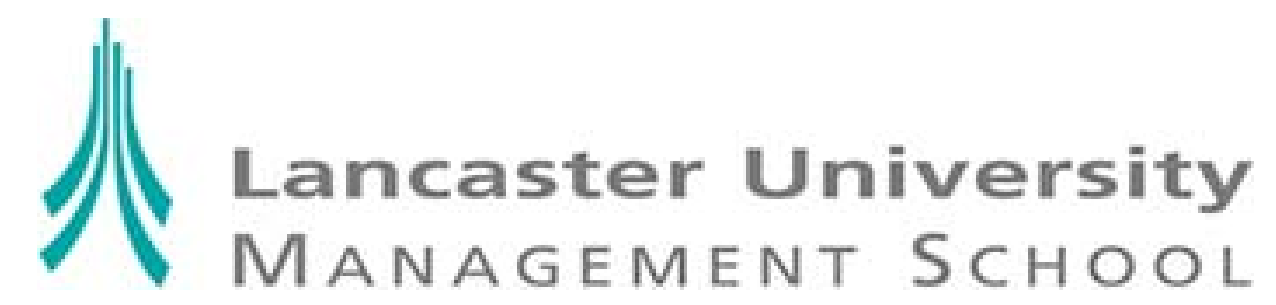

\author{
Lancaster University Management School \\ Working Paper \\ 2004/055
}

\title{
DARA and DRRA option bounds from concurrently expiring options
}

\author{
Huang James \\ The Department of Accounting and Finance \\ Lancaster University Management School \\ Lancaster LA1 4YX \\ UK \\ CHuang James \\ All rights reserved. Short sections of text, not to exceed \\ two paragraphs, may be quoted without explicit permission, \\ provided that full acknowledgement is given.
}

The LUMS Working Papers series can be accessed at http://www.lums.co.uk/publications/

LUMS home page: http://www.lums.lancs.ac.uk/ 


\title{
DARA and DRRA Option Bounds from
}

\section{Concurrently Expiring Options}

\author{
James Huang*
}

November 11, 2004

${ }^{*}$ Department of Accounting and Finance, Lancaster University, UK. LA1 4YX. Tel: +(44) 1524 593633, Fax: +(44) 1524 847321, Email: James.huang@lancaster.ac.uk. 


\begin{abstract}
In this paper we derive option bounds from concurrently expiring options assuming the representative investor has decreasing absolute \{relative risk aversion. We show that given the prices of the underlying stock and $n$ concurrently expiring options, the DARA $\{$ DRRA $\}$ option bound is given by a representative investor who has piecewise constant absolute \{relative $\}$ risk aversion. We also derive option bounds from concurrently expiring option prices assuming the representative investor has decreasing and bounded absolute $\{$ relative $\}$ risk aversion.
\end{abstract}

Keywords: Option bounds, Option pricing, DARA, DRRA.

JEL Classification Numbers: G13. 


\section{Introduction}

Since the works of Perrakis and Ryan (1984), Ritchken (1985), and Levy (1985) on option bounds, there has been good progress in this area. Ritchken and Kuo (1989) derived the $n$th order stochastic dominance option bounds. They also made good efforts on the DARA option bounds. ${ }^{1}$ Basso and Pianca (1997) continued the work on the DARA option bounds while Mathur and Ritchken (2000) finally obtained the result. Mathur and Ritchken found that the lower DARA $\{$ DRRA $\}$ option bound is given by a pricing representative investor who has CARA $\{$ CRRA $\}$ while the upper bound is the same as the $n$th order stochastic dominance option bound. ${ }^{2}$ Huang (2004) derived option bounds assuming the pricing representative investor has bounded relative risk aversion.

To further improve stochastic dominance option bounds Ryan (2003) suggested to use observed option prices. Ryan tightened the second stochastic dominance bounds by using one observed option price at a time. Huang (2004b) used a new method to further improve second order stochastic dominance option bounds using more than one observed prices of options and discussed the second order arbitrage opportunities in the markets of concurrently expiring options. The method was presented by Huang (2004a) to deal with option bound problems, which takes the advantage of the distinctive feature of options' payoff

\footnotetext{
${ }^{1}$ DARA $\{$ DRRA $\}$ denotes decreasing absolute \{relative $\}$ risk aversion.

${ }^{2}$ CARA $\{$ CRRA $\}$ denotes constant absolute \{relative $\}$ risk aversion.
} 
functions. Using the same methodology, Huang (2004c) improved higher order stochastic dominance option bounds from the observed prices of options.

In this paper we also use this method to improve the results on DARA \{DRRA $\}$ option bounds by using observed prices of concurrently expiring options. We derive DARA (DRRA) option bounds from observed concurrently expiring options. We show that given the prices of a unit zero-coupon bond, the underlying stock, and $n$ concurrently expiring options, the DARA $\{$ DRRA $\}$ option bound is given by a (pricing) representative investor who has piecewise CARA $\{\text { CRRA }\}^{3}$

We also derive option bounds from concurrently expiring options assuming the (pricing) representative investor has decreasing and bounded absolute \{relative risk aversion. In this case assuming the representative investor's absolute (relative) risk aversion is bounded above and below, given $n$ observed options, the upper (lower) option bound is given by a (pricing) representative investor who has $(n+2)$-segmented piecewise constant absolute $\{$ relative $\}$ risk aversion.

This paper is also related to the important works by Cochrane and SaaRequejo (2000) and Bernardo and Ledoit (2000) who presented different approaches to option bounds. Cochrane and Saa-Requejo derived option bounds using restrictions on the volatility of the pricing kernel, while Bernardo and Ledoit (2000) derived option bounds using restrictions on the deviation of the

\footnotetext{
${ }^{3}$ See, for example, Rubinstein (1976) and Brennan (1979) about a representative investor; see Benninga and Mayshar (2000) about a pricing representative investor.
} 
pricing kernel from a benchmark pricing kernel.

The structure of the paper is as follows: In Section 1 we introduce the assumptions. In Section 2 we deal with the case where there is one observed option. In Section 3 we deal with the case where there are two observed options. In Section 4 we deal with the general case where there are $n$ observed options. The final section concludes the paper.

\section{The DARA \{DRRA\} Assumption}

We assume that there is only one share of a stock in an economy on which option contracts are written. The price of the stock at time $t$ is denoted by $S_{t}$. Let $u(x)$ be the pricing representative investor's utility function. As is well known, the pricing kernel for the contingent claims on the stock is equal to the discounted marginal utility of wealth of the pricing representative investor. ${ }^{4}$ That is

$$
\phi\left(S_{t}\right)=\frac{u^{\prime}\left(S_{t}\right)}{E u^{\prime \prime\left(S_{t}\right)}} B_{0}
$$

where $\phi\left(S_{t}\right)$ denotes the pricing kernel and $B_{0}$ is the time 0 value of a unit zero coupon bond. ${ }^{5}$

Thus we have

$$
S_{0}=B_{0} E\left(\phi\left(S_{t}\right) S_{t}\right)
$$

Denote time $t$ value of a contingent claim by $c\left(S_{t}\right)$, which is dependent on $S_{t}$;

denote its time 0 value by $c_{0}$. Then we have $c_{0}=B_{0} E\left(\phi\left(S_{t}\right) c\left(S_{t}\right)\right)$, which is

\footnotetext{
${ }^{4}$ See, for example, Rubinstein (1976), Brennan (1979), and Benninga and Mayshar (2000).

${ }^{5}$ As there is only one share of the stock the pricing representative investor's wealth is equal to the stock price $S$.
} 
obviously dependent on $S_{0}$.

It is generally accepted that investors have decreasing absolute risk aversion or DARA. This implies

$$
\frac{d R\left(S_{t}\right)}{d S_{t}}<0
$$

where $R\left(S_{t}\right)=-u^{\prime \prime}\left(S_{t}\right) / u^{\prime}\left(S_{t}\right)$ is the pricing representative investor's absolute risk aversion.

Many people also believe that investors have decreasing relative risk aversion or DRRA. This implies

$$
\frac{d \gamma\left(S_{t}\right)}{d S_{t}}<0
$$

where $\gamma\left(S_{t}\right)=S R\left(S_{t}\right)$ is the pricing representative investor's relative absolute risk aversion.

Note we also have $R\left(S_{t}\right)=-\phi^{\prime}\left(S_{t}\right) / \phi\left(S_{t}\right)$ and $\gamma\left(S_{t}\right)=-S \phi^{\prime}\left(S_{t}\right) / \phi\left(S_{t}\right)$.

In this paper we are going to derive option bounds under the DARA $\{$ DRRA $\}$ assumption. To do this we first solve a similar but more general problem in which we assume that the representative investor's absolute/relative risk aversion is not only decreasing but also bounded from below and above. We will show in this paper that under this condition, the option bounds are given by a pricing representative investor who has piecewise constant absolute/relative risk aversion, where the number of segments of the risk aversion depends on the number of observed option prices.

Moreover, we will see that for an even number of observed option prices the risk aversion of the pricing representative investor who gives the option bounds has a certain pattern while for an odd number of observed option prices the risk 
aversion of the pricing representative investor who gives option bounds has a different pattern. Thus in order to explain the solutions more clearly we start with the case where we have only one observed option price then continue with the case where we have two observed options. Building on the above two cases we explore the general case where we have $n$ observed options.

Before we proceed we first make an explanation on the way we present our results. Note we find that the cases of DARA and DRRA can be dealt with in the same way. Thus for brevity we present the results on DARA and DRRA in a unified way. When doing so, anything corresponding to DRRA (which is parallel to something corresponding to DARA) is put in brackets " \{\} ".

\section{With One Observed Option}

Assume that the pricing representative investor's absolute \{relative\} risk aversion is decreasing and bounded above by $\bar{R}\{\bar{\gamma}\}$ and below by $\underline{R}\{\underline{\gamma}\}$. Assume we know the current value of the unit zero coupon bond $B_{0}$ and the spot stock price $S_{0}$. We now derive the option bounds. Before we proceed, we first introduce two lemmas.

Lemma 1 (FSS (1999)) Assume two pricing kernels give the same stock price. If they intersect twice, then the pricing kernel with fatter tails gives higher prices of convex-payoff contingent claims written on the stock.

Proof: See Huang (2004a) or FSS (1999). 
Lemma 2 Assume two pricing kernels give the same prices of the underlying stock and an option with strike price $K$. If they intersect three times, then the pricing kernel with fatter left tail will give higher [lower] prices for all options with strike prices below [above] $K$ than the other.

Proof: See Huang (2004a).

Proposition 1 Assume the pricing representative investor's absolute \{relative\} risk aversion is decreasing and bounded above by $\bar{R}\{\bar{\gamma}\}$ and below by $\underline{R}\{\underline{\gamma}\}$. Assume the prices of a unit zero-coupon bond, the underlying stock, and an option on the stock with strike price $K$ are $B_{0}, S_{0}$, and $c_{K 0}$ respectively.

- The upper bound for an option with a strike price below $K$ is given by the pricing kernel $\phi_{1}^{* *}$ corresponding to the pricing representative investor who has two-segmented piecewise constant absolute $\{$ relative $\}$ risk aversion. More precisely, his absolute $\{$ relative $\}$ risk aversion is equal to $\bar{R}$ $\{\bar{\gamma}\}$ for $S_{t}<s$ and $R^{* *}\left\{\gamma^{* *}\right\}$ for $S_{t}>s^{* *}$, where $R^{* *}\left\{\gamma^{* *}\right\}$ and $s^{* *}$ are decided by the underlying stock price and the observed option price. That is,

$$
\phi_{1}^{* *}(x)=\left\{\begin{array}{rcr}
a e^{-\bar{R} x}, & \left\{a x^{-\bar{\gamma}}\right\}, & \text { for } x<s^{* *} \\
a e^{\left(R^{* *}-\bar{R}\right) s^{* *}} e^{-R^{* *} x}, & \left\{a\left(s^{* *}\right)^{\gamma^{* *}-\bar{\gamma}} x^{-\gamma^{* *}}\right\}, & \text { for } x \geq s^{* *}
\end{array}\right.
$$
where $a$ and $s$ are decided such that $E\left(\phi_{1}^{* *}(x)\right)=1, E\left(S_{t} \phi_{1}^{* *}\left(S_{t}\right)\right) B_{0}=S_{0}$, and $E\left(c_{K}\left(S_{t}\right) \phi_{1}^{* *}\left(S_{t}\right)\right) B_{0}=c_{K 0}$.

- The lower bound for an option with a strike price below $K$ is given by the pricing kernel $\phi_{1}^{*}$ corresponding to the pricing representative investor 
who has two-segmented piecewise constant absolute $\{$ relative $\}$ risk aversion. More precisely,

$$
\phi_{1}^{*}(x)=\left\{\begin{array}{rcc}
a e^{-R^{*} x}, & \left\{a x^{-\gamma^{*}}\right\}, & \text { for } x<s^{*} \\
a e^{\left(\underline{R}-R^{*}\right) s^{*}} e^{-\underline{R} x}, & \left\{a\left(s^{*}\right)^{\left(\underline{\gamma}-\gamma^{*}\right)} x^{-\underline{\gamma}}\right\}, & \text { for } x \geq s^{*}
\end{array}\right.
$$

where $a, R^{*}\left\{\gamma^{*}\right\}$, and $s^{*}$ are decided such that $E\left(\phi_{1}^{*}\left(S_{t}\right)\right)=1, E\left(S_{t} \phi^{*}\left(S_{t}\right)\right)$ $B_{0}=S_{0}$, and $E\left(c_{K}\left(S_{t}\right) \phi_{1}^{*}\left(S_{t}\right)\right) B_{0}=c_{K 0}$.

- The lower (upper) bound for an option with a strike price above $K$ is given by the pricing kernel $\phi_{1}^{* *}\left(S_{t}\right)\left(\phi_{1}^{*}\left(S_{t}\right)\right)$.

Proof: From Lemma 2 we need only prove that the true pricing kernel intersects $\phi_{1}^{* *}(x)$ or $\phi_{1}^{*}(x)$ exactly three times and then examine which one has a fatter left tail.

We first examine $\phi_{1}^{* *}(x)$. Note its corresponding pricing representative investor has two-segmented piecewise constant absolute \{relative $\}$ risk aversion. More precisely, his absolute $\{$ relative $\}$ risk aversion is equal to $\bar{R} \quad\{\bar{\gamma}\}$ for $S_{t}<s$ and $R^{* *}\left\{\gamma^{* *}\right\}$ for $S_{t}>s^{* *}$. Obviously we must have $R^{* *} \in(\underline{R}, \bar{R})$ $\left\{\gamma^{* *} \in(\underline{\gamma}, \bar{\gamma})\right\}$. Otherwise the true pricing kernel will intersect $\phi_{1}^{* *}$ at most twice. In this case, applying Lemma 1, we find that the two pricing kernels cannot give the same observed option price. From this, we can immediately conclude that the true pricing kernel intersects $\phi_{1}^{* *}$ exactly three times. It is not difficult to verify that $\phi_{1}^{* *}$ has fatter left tail. For $\phi_{1}^{*}(x)$ the proof is similar. Q.E.D.

Corollary 1 Assume the pricing representative investor's absolute \{relative\} 
risk aversion is decreasing. Assume the prices of a unit zero-coupon bond, the underlying stock, and an option on the stock with strike price $K$ are $B_{0}, S_{0}$, and $c_{K 0}$ respectively.

Then the upper bound for options with strike prices below $K$ is given by the pricing kernel $\varphi_{1}^{* *}\left(S_{t}\right)=a \frac{\delta\left(S_{t}\right)}{p\left(S_{t}\right)}+b e^{-R^{* *} S_{t}}, \quad\left\{=a \frac{\delta\left(S_{t}\right)}{p\left(S_{t}\right)}+b S_{t}^{-\gamma^{* *}}\right\}$, where $p\left(S_{t}\right)$ is the probability density function, $\delta\left(S_{t}\right)$ is the Dirac function, and $a, b$, and $R^{* *}\left\{\gamma^{* *}\right\}$ are decided such that $E\left(\varphi_{1}^{* *}\left(S_{t}\right)\right)=1$, $E\left(S_{t} \varphi_{1}^{* *}\left(S_{t}\right)\right) B_{0}=S_{0}$, and $E\left(c_{K}\left(S_{t}\right) \varphi_{1}^{* *}\left(S_{t}\right)\right) B_{0}=c_{K 0}$.

- The lower bound for options with strike prices below $K$ is given by the pricing kernel

$$
\varphi_{1}^{*}(x)=\left\{\begin{array}{rrr}
a e^{-R^{*} x}, & \left\{a x^{-\gamma^{*}}\right\}, & \text { for } x<s^{*} \\
a e^{-R^{*} s^{*}}, & \left\{a s^{*-\gamma^{*}}\right\}, & \text { for } x \geq s^{*}
\end{array}\right.
$$

where $a, s^{*}$, and $R^{*}\left\{\gamma^{*}\right\}$ are decided such that $E\left(\varphi_{1}^{*}\left(S_{t}\right)\right)=1, E\left(S_{t} \varphi_{1}^{*}\left(S_{t}\right)\right)$ $B_{0}=S_{0}$, and $E\left(c_{K}\left(S_{t}\right) \varphi_{1}^{*}\left(S_{t}\right)\right) B_{0}=c_{K 0}$.

- The lower (upper) bound for options with strike prices above $K$ is given by the pricing kernel $\varphi_{l}^{* *}\left(S_{t}\right)\left(\varphi_{1}^{*}\left(S_{t}\right)\right)$.

Proof: Let $\bar{R} \rightarrow+\infty$ and $\underline{R} \rightarrow 0 \quad\{\bar{\gamma} \rightarrow+\infty$ and $\underline{\gamma} \rightarrow 0\}$; applying Proposition 1, we immediately obtain the result. Q.E.D.

\section{With Two Observed Options}

Lemma 3 Assume two pricing kernels give the same prices of the underlying stock and two options with strike prices $K_{1}$ and $K_{2}$, where $K_{1}<K_{2}$. If they 
intersect four times, then the pricing kernel with fatter left tail will give higher (lower) prices for options with strike prices outside (inside) $\left(K_{1}, K_{2}\right)$.

Proof: See Huang (2004a).

Proposition 2 Assume the pricing representative investor's absolute \{relative\} risk aversion is decreasing and bounded above by $\bar{R}\{\bar{\gamma}\}$ and below by $\underline{R}\{\underline{\gamma}\}$. Assume the price of a unit bond is $B_{0}$, the underlying stock price is $S_{0}$, and the prices of two options with strike prices $K_{1}$ and $K_{2}$ are $c_{10}$ and $c_{20}$ respectively, where $K_{1}<K_{2}$.

- Then the upper bound for options with strike prices below $K_{1}$ or above $K_{2}$ is given by the pricing kernel $\phi_{2}^{* *}(x)$ corresponding to the pricing representative investor who has three-segmented piecewise constant absolute \{relative\} risk aversion. More precisely, his absolute $\{$ relative $\}$ risk aversion is equal to $\bar{R}\{\bar{\gamma}\}$ for $S_{t}<s_{1}^{* *}$, and $R^{* *}\left\{\gamma^{* *}\right\}$ for $S_{t} \in\left(s_{1}^{* *}, s_{2}^{* *}\right)$, and $\underline{R}\{\underline{\gamma}\}$ for $S_{t}>s_{2}^{* *}$, where $s_{1}^{* *}, s_{1}^{* *}$, and $R^{* *}\left\{\gamma^{* *}\right\}$ are decided by the underlying stock price and the two option prices. That is, $\phi_{2}^{* *}(x)=$

$$
\begin{array}{cc}
a e^{-\bar{R} x}, \quad\left\{a x^{-\bar{\gamma}}\right\}, & x<s_{1}^{* *} \\
a e^{\left(R^{* *}-\bar{R}\right) s_{1}^{* *}} e^{-R^{* *} x}, \quad\left\{a\left(s_{1}^{* *}\right)^{\gamma^{* *}-\bar{\gamma}} x^{-\gamma^{* *}}\right\}, & x \in\left(s_{1}^{* *}, s_{2}^{* *}\right) \\
a e^{\left(R^{* *}-\bar{R}\right) s_{1}^{* *}} e^{\left(\underline{R}-R^{* *}\right) s_{2}^{* *}} e^{-\underline{R} x},\left\{a\left(s_{1}^{* *}\right)^{\gamma^{* *}-\bar{\gamma}}\left(s_{2}^{* *}\right) \underline{\gamma-\gamma^{* *}} x^{-\underline{\gamma}}\right\}, & x>s_{2}^{* *} \\
\text { where } a, s_{1}^{* *}, s_{1}^{* *}, \text { and } R^{* *}\left\{\gamma^{* *}\right\} \text { are decided such that } E\left(\phi_{2}^{* *}(x)\right)=1, \\
E\left(S_{t} \phi_{2}^{* *}\left(S_{t}\right)\right) B_{0}=S_{0}, E\left(c_{1}\left(S_{t}\right) \phi_{2}^{* *}\left(S_{t}\right)\right) B_{0}=c_{10}, \text { and } E\left(c_{2}\left(S_{t}\right) \phi_{2}^{* *}\left(S_{t}\right)\right) \\
B_{0}=c_{20} .
\end{array}
$$

- The lower bound for options with strike prices below $K_{1}$ or above $K_{2}$ is 
given by the pricing kernel $\phi_{2}^{*}(x)$ corresponding to the pricing representative investor who has two-segmented piecewise constant absolute $\{$ relative $\}$ risk aversion. More precisely,

$$
\phi_{2}^{*}(x)=\left\{\begin{array}{rcc}
a e^{-R_{1}^{*} x}, & \left\{a x^{-\gamma_{1}^{*}}\right\}, & \text { for } x<s^{*} \\
a e^{\left(R_{2}^{*}-R_{1}^{*}\right) s^{*}} e^{-R_{2}^{*} x}, & \left\{a\left(s^{*}\right)^{\gamma_{2}^{*}-\gamma_{1}^{*}} x^{-\gamma_{2}^{*}}\right\}, & \text { for } x \geq s^{*}
\end{array}\right.
$$

where $a, s^{*}, R_{1}^{*}\left\{\gamma_{1}^{*}\right\}$, and $R_{2}^{*}\left\{\gamma_{2}^{*}\right\}$ are decided such that $E\left(\phi_{2}^{*}(x)\right)=1$, $E\left(S_{t} \phi_{2}^{*}\left(S_{t}\right)\right) B_{0}=S_{0}, E\left(c_{1}\left(S_{t}\right) \phi_{2}^{*}\left(S_{t}\right)\right) B_{0}=c_{10}$, and $E\left(c_{2}\left(S_{t}\right) \phi_{2}^{*}\left(S_{t}\right)\right) B_{0}=$ $c_{20}$.

- The lower (upper) bound for options with strike prices between $K_{1}$ and $K_{2}$ is given by the pricing kernel $\phi_{2}^{*}\left(S_{t}\right)\left(\phi_{2}^{* *}\left(S_{t}\right)\right)$.

Proof: From Lemma 3 we need only prove that the true pricing kernel intersects $\phi_{2}^{* *}(x)$ or $\phi_{2}^{*}(x)$ exactly four times and then examine which one has a fatter left tail.

We first examine $\phi_{2}^{* *}(x)$. Note its corresponding pricing representative investor has three-segmented piecewise constant absolute \{relative $\}$ risk aversion. More precisely, his absolute $\{$ relative $\}$ risk aversion is equal to $\bar{R}\{\bar{\gamma}\}$ for $S_{t}<s_{1}^{* *}$, and $R^{* *}\left\{\gamma^{* *}\right\}$ for $S_{t} \in\left(s_{1}^{* *}, s_{2}^{* *}\right)$, and $\underline{R}\{\underline{\gamma}\}$ for $S_{t}>s_{2}^{* *}$. Obviously we must have $R^{* *} \in(\underline{R}, \bar{R}) \quad\left\{\gamma^{* *} \in(\underline{\gamma}, \bar{\gamma})\right\}$. Otherwise the true pricing kernel will intersect $\phi_{2}^{* *}$ at most three times. In this case, applying Lemma 2, we find that the two pricing kernels cannot give the same two observed option prices. From this, we can immediately conclude that the true pricing kernel intersects $\phi_{2}^{* *}$ exactly four times. It is not difficult to verify that $\phi_{2}^{* *}$ has fatter left tail. For $\phi_{2}^{*}(x)$ the proof is similar. Q.E.D. 
Corollary 2 Assume the price of a unit bond is $B_{0}$, the underlying stock price is $S_{0}$, and the prices of two options with strike prices $K_{1}$ and $K_{2}$ are $c_{10}$ and $c_{20}$ respectively, where $K_{1}<K_{2}$.

Then the upper bound for options with strike prices below $K_{1}$ or above $K_{2}$ is given by the pricing kernel $\varphi_{2}^{* *}\left(S_{t}\right)=a_{0} \frac{\delta\left(S_{t}\right)}{p\left(S_{t}\right)}+f^{* *}\left(S_{t}\right)$, where $p\left(S_{t}\right)$ is the probability density function, $\delta\left(S_{t}\right)$ is the Dirac function,

$$
\begin{aligned}
& f^{* *}(x)=\left\{\quad a_{1} e^{-R^{* *} x}, \quad\left\{a_{1} x^{-\gamma^{* *}}\right\}, \quad \text { for } x<s^{* *}\right. \\
& a_{1} e^{-R^{* *} s^{* *}}, \quad\left\{a_{1} s^{* *-\gamma^{* *}}\right\}, \quad \text { for } x \geq s^{*}
\end{aligned}
$$

and $a_{0}, a_{1}, s^{* *}$, and $R^{* *}\left\{\gamma^{* *}\right\}$ are decided such that $E\left(\varphi_{2}^{* *}\left(S_{t}\right)\right)=1$, $E\left(S_{t} \varphi_{2}^{* *}\left(S_{t}\right)\right) B_{0}=S_{0}$, and $E\left(c_{1}\left(S_{t}\right) \varphi_{2}^{* *}\left(S_{t}\right)\right) B_{0}=c_{10}$, and $E\left(c_{2}\left(S_{t}\right) \varphi_{2}^{* *}\left(S_{t}\right)\right)$ $B_{0}=c_{20}$.

- The lower bound for options with strike prices below $K_{1}$ or above $K_{2}$ is given by the pricing kernel $\varphi_{2}^{*}(x)=\phi_{2}^{*}(x)$.

- The lower (upper) bound for options with strike prices between $K_{1}$ and $K_{2}$ is given by the pricing kernel $\varphi_{2}^{* *}\left(S_{t}\right)\left(\varphi_{2}^{*}\left(S_{t}\right)\right)$.

Proof: Let $\bar{R} \rightarrow+\infty$ and $\underline{R} \rightarrow 0 \quad\{\bar{\gamma} \rightarrow+\infty$ and $\underline{\gamma} \rightarrow 0\}$; applying Proposition 2, we immediately obtain the result. Q.E.D.

\section{The General Case}

Lemma 4 Assume two pricing kernels give the same prices of the underlying stock and options with strike prices $K_{1}, K_{2}, \ldots, K_{n}$, where $K_{1}<K_{2}<\ldots<K_{n}$. 
Let $K_{0}=0$ and $K_{n+1}=+\infty$. If the two pricing kernels intersect $n+2$ times then the one with fatter left tail will give higher (lower) prices for all options with strike prices between $\left(K_{2 i-2}, K_{2 i-1}\right)\left(\left(K_{2 i-1}, K_{2 i}\right)\right), i=1,2, \ldots$.

Proof: See Huang (2004a).

Proposition 3 Assume the pricing representative investor's absolute $\{$ relative $\}$ risk aversion is decreasing and bounded above by $\bar{R}\{\bar{\gamma}\}$ and below by $\underline{R}\{\underline{\gamma}\}$. Assume the price of a unit bond is $B_{0}$, the underlying stock price is $S_{0}$, and the prices of $n$ options with strike prices $K_{1}, K_{2}, \ldots, K_{n}$ are $c_{10}, c_{20}, \ldots$, and $c_{n 0}$ respectively, where $K_{1}<K_{2}<\ldots<K_{n}$. Let $K_{0}=0$ and $K_{n+1}=+\infty$.

- Assume $n$ is odd. Let $m=(n+1) / 2$.

- Then the upper bound for options with strike prices between $\left(K_{2 i-2}\right.$, $\left.K_{2 i-1}\right), i=1,2, \ldots$, is given by the pricing kernel $\phi_{n}^{* *}$ corresponding to the pricing representative investor who has $(m+1)$-segmented piecewise constant absolute $\{$ relative $\}$ risk aversion. More precisely, his absolute $\{$ relative $\}$ risk aversion is equal to $\bar{R}\{\bar{\gamma}\}$ for $S_{t}<$ $s_{1}^{* *}, R_{1}^{* *}\left\{\gamma_{1}^{* *}\right\}$ for $S_{t} \in\left(s_{1}^{* *}, s_{2}^{* *}\right), \ldots, R_{m-1}^{* *}\left\{\gamma_{m-1}^{* *}\right\}$ for $S_{t} \in$ $\left(s_{m-1}^{* *}, s_{m}^{* *}\right), R_{m}^{* *}\left\{\gamma_{m}^{* *}\right\}$ for $S_{t}>s_{m}^{* *}$, where $s_{1}^{* *}, \ldots, s_{m}^{* *}, R_{1}^{* *}\left\{\gamma_{1}^{* *}\right\}$ $\ldots$, and $R_{m}^{* *}\left\{\gamma_{m}^{* *}\right\}$ are decided by the underlying stock price and the 
observed $n$ option prices. That is, $\phi_{n}^{* *}(x)=$

$$
\begin{aligned}
& a e^{-\bar{R} x}, \quad x<s_{1}^{* *} \\
& a e^{\left(R_{1}^{* *}-\bar{R}\right) s_{1}^{* *}} e^{-R_{1}^{* *} x}, \quad x \in\left(s_{1}^{* *}, s_{2}^{* *}\right) \\
& a e^{\left(R_{1}^{* *}-\bar{R}\right) s_{1}^{* *}} \ldots e^{\left(R_{m-1}^{* *}-R_{m-2}^{* *}\right) s_{m-1}^{* *}} e^{\left(R_{m}^{* *}-R_{m-1}^{* *}\right) s_{m}^{* *}} e^{-R_{m}^{* *} x}, \quad x>s_{m}^{* *} \\
& \left\{a x^{-\bar{\gamma}}, \quad x<s_{1}^{* *}\right\} \\
& \left\{a\left(s_{1}^{* *}\right)^{\gamma_{1}^{* *}-\bar{\gamma}} x^{-\gamma_{1}^{* *}}, \quad x \in\left(s_{1}^{* *}, s_{2}^{* *}\right)\right\} \\
& \left\{a\left(s_{1}^{* *}\right)^{\gamma_{1}^{* *}-\bar{\gamma}} \ldots\left(s_{m-1}^{* *}\right)^{\gamma_{m-1}^{* *}-\gamma_{m-2}^{* *}}\left(s_{m}^{* *}\right)^{\gamma_{m}^{* *}-\gamma_{m-1}^{* *}} x^{-\gamma_{m}^{* *}}, \quad x>s_{m}^{* *}\right\}
\end{aligned}
$$

where $a, s_{1}^{* *}, \ldots, s_{m}^{* *}, R_{1}^{* *}\left\{\gamma_{1}^{* *}\right\}, \ldots$, and $R_{m}^{* *}\left\{\gamma_{m}^{* *}\right\}$ are decided such that $E\left(\phi_{n}^{* *}(x)\right)=1, E\left(S_{t} \phi_{n}^{* *}\left(S_{t}\right)\right) B_{0}=S_{0}$, and $E\left(c_{i}\left(S_{t}\right) \phi_{n}^{* *}\left(S_{t}\right)\right) B_{0}=$ $c_{i 0}, i=1,2, \ldots, n$.

- The lower bound for options with strike prices between $\left(K_{2 i-2}, K_{2 i-1}\right)$, $i=1,2, \ldots$, is given by the pricing kernel $\phi_{n}^{*}$ corresponding to the pricing representative investor who has $(m+1)$-segmented piecewise constant absolute $\{$ relative\} risk aversion. More precisely, his absolute \{relative $\}$ risk aversion is equal to $R_{1}^{*}\left\{\gamma_{1}^{*}\right\}$ for $S_{t}<s_{1}^{*}, R_{2}^{*}\left\{\gamma_{2}^{*}\right\}$ for $S_{t} \in\left(s_{1}^{*}, s_{2}^{*}\right), \ldots, R_{m}^{*} \quad\left\{\gamma_{m}^{*}\right\}$ for $S_{t} \in\left(s_{m-1}^{*}, s_{m}^{*}\right), \underline{R}\{\underline{\gamma}\}$ for $S_{t}>s_{m}^{*}$, where $s_{1}^{*}, \ldots, s_{m}^{*}, R_{1}^{*}\left\{\gamma_{1}^{*}\right\}, \ldots$, and $R_{m}^{*}\left\{\gamma_{m}^{*}\right\}$ are decided by the underlying stock price and the observed $n$ option prices. That 
$i s, \phi_{n}^{*}(x)=$

$$
\begin{aligned}
& a e^{-R_{1}^{*} x}, \quad x<s_{1}^{*} \\
& a e^{\left(R_{2}^{*}-R_{1}^{*}\right) s_{1}^{*}} e^{-R_{2}^{*} x}, \quad x \in\left(s_{1}^{*}, s_{2}^{*}\right) \\
& a e^{\left(R_{2}^{*}-R_{1}^{*}\right) s_{1}^{*}} \ldots e^{\left(R_{m}^{*}-R_{m-1}^{*}\right) s_{m-1}^{*}} e^{\left(\underline{R}-R_{m}^{*}\right) s_{m}^{*}} e^{-\underline{R} x}, \quad x>s_{m}^{*} \\
& \left\{a x^{-\gamma_{1}^{*}}, \quad x<s_{1}^{*}\right\} \\
& \left\{a\left(s_{1}^{*}\right)^{\gamma_{2}^{*}-\gamma_{1}^{*}} x^{-\gamma_{2}^{*}}, \quad x \in\left(s_{1}^{*}, s_{2}^{*}\right)\right\}
\end{aligned}
$$

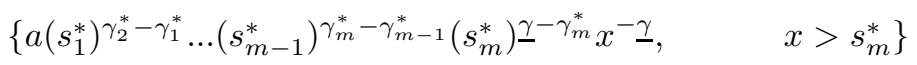

where $a, s_{1}^{*}, \ldots, s_{m}^{*}, R_{1}^{*}\left\{\gamma_{1}^{*}\right\}, \ldots$, and $R_{m}^{*}\left\{\gamma_{m}^{*}\right\}$ are decided such that $E\left(\phi_{n}^{*}(x)\right)=1, E\left(S_{t} \phi_{n}^{*}\left(S_{t}\right)\right) B_{0}=S_{0}$, and $E\left(c_{i}\left(S_{t}\right) \phi_{n}^{*}\left(S_{t}\right)\right) B_{0}=c_{i 0}$, $i=1,2, \ldots, n$.

- The lower (upper) bound for options with strike prices between $\left(K_{2 i-1}\right.$, $\left.K_{2 i}\right), i=1,2, \ldots$, is given by the pricing kernel $\phi_{n}^{* *}\left(S_{t}\right)\left(\phi_{n}^{*}\left(S_{t}\right)\right)$.

- Assume $n$ is even. Let $m=n / 2$.

- Then the upper bound for options with strike prices between $\left(K_{2 i-2}\right.$, $\left.K_{2 i-1}\right), i=1,2, \ldots$, is given by the pricing kernel $\phi_{n}^{* *}(x)$ corresponding to the pricing representative investor who has $(m+2)$-segmented piecewise constant absolute $\{$ relative $\}$ risk aversion. More precisely, his absolute $\{$ relative $\}$ risk aversion is equal to $\bar{R}\{\bar{\gamma}\}$ for $S_{t}<$ $s_{1}^{* *}$, and $R_{1}^{* *}\left\{\gamma_{1}^{* *}\right\}$ for $S_{t} \in\left(s_{1}^{* *}, s_{2}^{* *}\right), \ldots, R_{m}^{* *}\left\{\gamma_{m}^{* *}\right\}$ for $S_{t} \in$ 
$\left(s_{m}^{* *}, s_{m+1}^{* *}\right)$, and $\underline{R}\{\underline{\gamma}\}$ for $S_{t}>s_{m+1}^{* *}$, , where $s_{1}^{* *}, \ldots, s_{m+1}^{* *}$, $R_{1}^{* *}\left\{\gamma_{1}^{* *}\right\}, \ldots$, and $R_{m}^{* *}\left\{\gamma_{m}^{* *}\right\}$ are decided by the underlying stock price and the $n$ option prices. That is, $\phi_{n}^{* *}(x)=$

$$
\begin{aligned}
& a e^{-\bar{R} x}, \quad x<s_{1}^{* *} \\
& a e^{\left(R_{1}^{* *}-\bar{R}\right) s_{1}^{* *}} e^{-R_{1}^{* *} x}, \quad s_{1}^{* *}<x<s_{2}^{* *} \\
& a e^{\left(R_{1}^{* *}-\bar{R}\right) s_{1}^{* *}} \ldots e^{\left(R_{m}^{* *}-R_{m-1}^{* *}\right) s_{m}^{* *}} e^{-R_{m}^{* *} x}, \quad s_{m}^{* *}<x<s_{m+1}^{* *} \\
& a e^{\left(R_{1}^{* *}-\bar{R}\right) s_{1}^{* *}} \ldots e^{\left(R_{m}^{* *}-R_{m-1}^{* *}\right) s_{m}^{* *}} e^{\left(\underline{R}-R_{m}^{* *}\right) s_{m+1}^{* *}} e^{-\underline{R} x}, \quad x>s_{m+1}^{* *} \\
& \left\{a x^{-\bar{\gamma}}, \quad x<s_{1}^{* *}\right\} \\
& \left\{a\left(s_{1}^{* *}\right)^{\gamma_{1}^{* *}-\bar{\gamma}} x^{-\gamma_{1}^{* *}}, \quad s_{1}^{* *}<x<s_{2}^{* *}\right\} \\
& \left\{a\left(s_{1}^{* *}\right)^{\gamma_{1}^{* *}-\bar{\gamma}} \ldots\left(s_{m}^{* *}\right)^{\gamma_{m}^{* *}-\gamma_{m-1}^{* *}} x^{-\gamma_{m}^{* *}}, \quad s_{m}^{* *}<x<s_{m+1}^{* *}\right\} \\
& \left\{a\left(s_{1}^{* *}\right)^{\gamma_{1}^{* *}-\bar{\gamma}} \ldots\left(s_{m}^{* *}\right)^{\gamma_{m}^{* *}-\gamma_{m-1}^{* *}}\left(s_{m+1}^{* *}\right){\underline{\gamma}-\gamma_{m}^{* *}}^{-\underline{\gamma}}, \quad x>s_{m+1}^{* *}\right\}
\end{aligned}
$$

where $a, s_{1}^{* *}, \ldots, s_{m+1}^{* *}, R_{1}^{* *}\left\{\gamma_{1}^{* *}\right\}, \ldots$, and $R_{m}^{* *}\left\{\gamma_{m}^{* *}\right\}$ are decided such that $E\left(\phi_{n}^{* *}\left(S_{t}\right)\right)=1, E\left(S_{t} \phi_{n}^{* *}\left(S_{t}\right)\right) B_{0}=S_{0}$, and $E\left(c_{i}\left(S_{t}\right) \phi_{n}^{* *}\left(S_{t}\right)\right)$ $B_{0}=c_{i 0}, i=1,2, \ldots, n$.

- The lower bound for options with strike prices between $\left(K_{2 i-2}, K_{2 i-1}\right)$, $i=1,2, \ldots$, is given by the pricing kernel $\left(\phi_{2}^{*}(x)\right)$ corresponding to the pricing representative investor who has $(m+1)$-segmented piecewise 
constant absolute $\{$ relative $\}$ risk aversion. More precisely, $\phi_{n}^{*}(x)=$

$$
\begin{aligned}
& a e^{-R_{1}^{*} x}, \quad x<s_{1}^{*} \\
& a e^{\left(R_{2}^{*}-R_{1}^{*}\right) s_{1}^{*}} e^{-R_{2}^{*} x}, \quad s_{1}^{*}<x<s_{2}^{*} \\
& a e^{\left(R_{2}^{*}-R_{1}^{*}\right) s_{1}^{*}} \ldots e^{\left(R_{m+1}^{*}-R_{m}^{*}\right) s_{m}^{*}} e^{-R_{m+1}^{*} x}, \quad x>s_{m}^{*} \\
& \left\{a x^{-\gamma_{1}^{*}}, \quad x<s_{1}^{*}\right\} \\
& \left\{a\left(s_{1}^{*}\right)^{\gamma_{2}^{*}-\gamma_{1}^{*}} x^{-\gamma_{2}^{*}}, \quad s_{1}^{*}<x<s_{2}^{*}\right\} \\
& \left\{a\left(s_{1}^{*}\right)^{\gamma_{2}^{*}-\gamma_{1}^{*}} \ldots\left(s_{m}^{*}\right)^{\gamma_{m+1}^{*}-\gamma_{m}^{*}} x^{-\gamma_{m+1}^{*}}, \quad x>s_{m}^{*}\right\}
\end{aligned}
$$

where $a, s_{1}^{*}, \ldots, s_{m}^{*}, R_{1}^{*}\left\{\gamma_{1}^{*}\right\}, \ldots$, and $R_{m+1}^{*}\left\{\gamma_{m+1}^{*}\right\}$ are decided such that $E\left(\phi_{n}^{*}(x)\right)=1, E\left(S_{t} \phi_{n}^{*}\left(S_{t}\right)\right) B_{0}=S_{0}$, and $E\left(c_{i}\left(S_{t}\right) \phi_{n}^{*}\left(S_{t}\right)\right)$ $B_{0}=c_{i 0}, i=1,2, \ldots, n$.

- The lower (upper) bound for options with strike prices between $\left(K_{2 i-1}\right.$, $\left.K_{2 i}\right), i=1,2, \ldots$, is given by the pricing kernel $\phi_{n}^{* *}\left(S_{t}\right)\left(\phi_{n}^{*}\left(S_{t}\right)\right)$.

Proof: From Lemma 4 we need only prove that the true pricing kernel intersects $\phi_{n}^{* *}(x)$ or $\phi_{n}^{*}(x)$ exactly $(n+2)$ times and then examine which one has a fatter left tail.

We first examine $\phi_{n}^{* *}(x)$. Note its corresponding to the pricing representative investor who has $(\mathrm{m}+1)$-segmented piecewise constant absolute relative risk aversion. More precisely, his absolute \{relative \} risk aversion is equal to $\bar{R}\{\bar{\gamma}\}$ for $S_{t}<s_{1}^{* *}, R_{1}^{* *}\left\{\gamma_{1}^{* *}\right\}$ for $S_{t} \in\left(s_{1}^{* *}, s_{2}^{* *}\right), \ldots, R_{m-1}^{* *}\left\{\gamma_{m-1}^{* *}\right\}$ for $S_{t} \in\left(s_{m-1}^{* *}, s_{m}^{* *}\right), R_{m}^{* *}\left\{\gamma_{m}^{* *}\right\}$ for $S_{t}>s_{m}^{* *}$ 
We must have $\bar{R}>R_{1}^{* *}>\ldots>R_{m-1}^{* *}>R_{m}^{* *}>\underline{R}\left\{\bar{\gamma}>\gamma_{1}^{* *}>\ldots>\gamma_{m-1}^{* *}>\right.$ $\gamma_{m}^{* *}>\underline{\gamma}$. Otherwise the true pricing kernel will intersect $\phi_{n}^{* *}$ at most three times. In this case, applying Lemma 4, we find that the two pricing kernels cannot give the same $n$ observed option prices. From this, we can immediately conclude that the true pricing kernel intersects $\phi_{n}^{* *}$ exactly $n+2$ times. It is not difficult to verify that $\phi_{n}^{* *}$ has fatter left tail. For $\phi_{n}^{*}(x)$ the proof is similar. Q.E.D.

Corollary 3 Assume the elasticity of the pricing kernel is decreasing in the underlying stock price. Assume the price of a unit bond is $B_{0}$, the underlying stock price is $S_{0}$, and the prices of $n$ options with strike prices $K_{1}, K_{2}, \ldots, K_{n}$ are $c_{10}, c_{20}, \ldots$, and $c_{n 0}$ respectively, where $K_{1}<K_{2}<\ldots<K_{n}$. Let $K_{0}=0$ and $K_{n+1}=+\infty$.

- Assume $n$ is odd. Let $m=(n+1) / 2$.

- Then the upper bound for options with strike prices between $\left(K_{2 i-2}\right.$, $\left.K_{2 i-1}\right), i=1,2, \ldots$, is given by the pricing kernel $\varphi_{n}^{* *}\left(S_{t}\right)=a_{0} \frac{\delta\left(S_{t}\right)}{p\left(S_{t}\right)}+$ $f_{n}^{* *}\left(S_{t}\right)$, where $p\left(S_{t}\right)$ is the probability density function, $\delta\left(S_{t}\right)$ is the Dirac function, and $f_{n}^{* *}(x)=$

$$
\begin{array}{cc}
a e^{-R_{1}^{* *} x}, & x<s_{2}^{* *} \\
a e^{\left(R_{2}^{* *}-R_{1}^{* *}\right) s_{2}^{* *}} e^{-R_{2}^{* *} x}, & s_{2}^{* *}<x<s_{3}^{* *} \\
\cdots \ldots & \\
a e^{\left(R_{2}^{* *}-R_{1}^{* *}\right) s_{2}^{* *}} \ldots e^{\left(R_{m}^{* *}-R_{m-1}^{* *}\right) s_{m}^{* *}} e^{-R_{m}^{* *} x}, & x>s_{m}^{* *}
\end{array}
$$




$$
\begin{array}{cc}
\left\{a x^{-\gamma_{1}^{* *}},\right. & \left.x<s_{2}^{* *}\right\} \\
\left\{a\left(s_{2}^{* *}\right)^{\gamma_{2}^{* *}-\gamma_{1}^{* *}} x^{-\gamma_{2}^{* *}},\right. & \left.s_{2}^{* *}<x<s_{3}^{* *}\right\} \\
\ldots \ldots & \\
\left\{a\left(s_{2}^{* *}\right)^{\gamma_{2}^{* *}-\gamma_{1}^{* *}} \ldots\left(s_{m}^{* *}\right)^{\gamma_{m}^{* *}-\gamma_{m-1}^{* *}} x^{-\gamma_{m}^{* *}},\right. & \left.x>s_{m}^{* *}\right\}
\end{array}
$$

where $a_{0}, a, s_{2}^{* *}, \ldots, s_{m}^{* *}, R_{1}^{* *}\left\{\gamma_{1}^{* *}\right\}, \ldots$ and $R_{m}^{* *}\left\{\gamma_{m}^{* *}\right\}$ are decided such that $E\left(\varphi_{n}^{* *}(x)\right)=1, E\left(S_{t} \varphi_{n}^{* *}\left(S_{t}\right)\right) B_{0}=S_{0}$, and $E\left(c_{i}\left(S_{t}\right) \varphi_{n}^{* *}\left(S_{t}\right)\right)$ $B_{0}=c_{i 0}, i=1,2, \ldots, n$.

- The lower bound for options with strike prices between $\left(K_{2 i-2}, K_{2 i-1}\right)$, $i=1,2, \ldots$, is given by the pricing kernel $\varphi_{n}^{*}(x)=$

$$
\begin{aligned}
& a e^{-R_{1}^{*} x}, \quad x<s_{1}^{*} \\
& a e^{\left(R_{2}^{*}-R_{1}^{*}\right) s_{1}^{*}} e^{-R_{2}^{*} x}, \quad x \in\left(s_{1}^{*}, s_{2}^{*}\right) \\
& a e^{\left(R_{2}^{*}-R_{1}^{*}\right) s_{1}^{*}} \ldots e^{\left(R_{m}^{*}-R_{m-1}^{*}\right) s_{m-1}^{*}} e^{-R_{m}^{*} s_{m}^{*}}, \quad x>s_{m}^{*} \\
& \left\{a x^{-\gamma_{1}^{*}}, \quad x<s_{1}^{*}\right\} \\
& \left\{a\left(s_{1}^{*}\right)^{\gamma_{2}^{*}-\gamma_{1}^{*}} x^{-\gamma_{2}^{*}}, \quad x \in\left(s_{1}^{*}, s_{2}^{*}\right)\right\}
\end{aligned}
$$

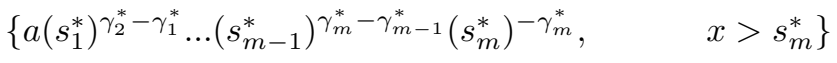

where $a, s_{1}^{*}, \ldots, s_{m}^{*}, R_{1}^{*}\left\{\gamma_{1}^{*}\right\}, \ldots$, and $R_{m}^{*}\left\{\gamma_{m}^{*}\right\}$ are decided such that $E\left(\phi_{n}^{*}(x)\right)=1, E\left(S_{t} \phi_{n}^{*}\left(S_{t}\right)\right) B_{0}=S_{0}$, and $E\left(c_{i}\left(S_{t}\right) \phi_{n}^{*}\left(S_{t}\right)\right) B_{0}=c_{i 0}$, $i=1,2, \ldots, n$.

- The lower (upper) bound for options with strike prices between $\left(K_{2 i-1}\right.$, $\left.K_{2 i}\right), i=1,2, \ldots$, is given by the pricing kernel $\varphi_{n}^{* *}\left(S_{t}\right)\left(\varphi_{n}^{*}\left(S_{t}\right)\right)$. 
- Assume $n$ is even. Let $m=n / 2$.

- Then the upper bound for options with strike prices between $\left(K_{2 i-2}\right.$, $\left.K_{2 i-1}\right), i=1,2, \ldots$, is given by the pricing kernel $\varphi_{n}^{* *}\left(S_{t}\right)=a_{0} \frac{\delta\left(S_{t}\right)}{p\left(S_{t}\right)}+$ $f_{n}^{* *}\left(S_{t}\right)$, where $p\left(S_{t}\right)$ is the probability density function, $\delta\left(S_{t}\right)$ is the Dirac function, and $f_{n}^{* *}(x)=$

$$
\begin{aligned}
& a e^{-R_{1}^{* *} x}, \quad x<s_{2}^{* *} \\
& a e^{\left(R_{2}^{* *}-R_{1}^{* *}\right) s_{2}^{* *}} e^{-R_{2}^{* *} x}, \quad s_{2}^{* *}<x<s_{3}^{* *} \\
& a e^{\left(R_{2}^{* *}-R_{1}^{* *}\right) s_{2}^{* *}} \ldots e^{\left(R_{m}^{* *}-R_{m-1}^{* *}\right) s_{m}^{* *}} e^{-R_{m}^{* *} x}, \quad s_{m}^{* *}<x<s_{m+1}^{* *} \\
& a e^{\left(R_{2}^{* *}-R_{1}^{* *}\right) s_{2}^{* *}} \ldots e^{\left(R_{m}^{* *}-R_{m-1}^{* *}\right) s_{m}^{* *}} e^{-R_{m}^{* *} s_{m+1}^{* *}}, \quad x>s_{m+1}^{* *} \\
& \left\{a x^{-\gamma_{1}^{* *},} \quad x<s_{2}^{* *}\right\} \\
& \left\{a\left(s_{2}^{* *}\right)^{\gamma_{2}^{* *}-\gamma_{1}^{* *}} x^{-\gamma_{2}^{* *},} \quad s_{2}^{* *}<x<s_{3}^{* *}\right\}
\end{aligned}
$$

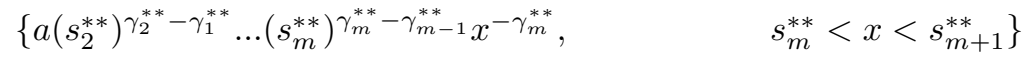

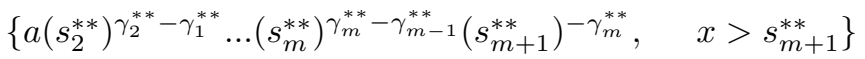

where $a_{0}, a, s_{2}^{* *}, \ldots, s_{m+1}^{* *}, R_{1}^{* *}\left\{\gamma_{1}^{* *}\right\}, \ldots$, and $R_{m}^{* *}\left\{\gamma_{m}^{* *}\right\}$ are decided such that $E\left(\varphi_{n}^{* *}\left(S_{t}\right)\right)=1, E\left(S_{t} \varphi_{n}^{* *}\left(S_{t}\right)\right) B_{0}=S_{0}$, and $E\left(c_{i}\left(S_{t}\right) \varphi_{n}^{* *}\left(S_{t}\right)\right)$ $B_{0}=c_{i 0}, i=1,2, \ldots, n$.

- The lower bound for options with strike prices between $\left(K_{2 i-2}, K_{2 i-1}\right)$, 


$$
\begin{aligned}
& i=1,2, \ldots, \text { is given by the pricing kernel } \varphi_{n}^{*}(x)= \\
& a e^{-R_{1}^{*} x}, \quad x<s_{1}^{*} \\
& a e^{\left(R_{2}^{*}-R_{1}^{*}\right) s_{1}^{*}} e^{-R_{2}^{*} x}, \quad s_{1}^{*}<x<s_{2}^{*} \\
& a e^{\left(R_{2}^{*}-R_{1}^{*}\right) s_{1}^{*}} \ldots e^{\left(R_{m+1}^{*}-R_{m}^{*}\right) s_{m}^{*}} e^{-R_{m+1}^{*} x}, \quad x>s_{m}^{*} \\
& \left\{a x^{-\gamma_{1}^{*}}, \quad x<s_{1}^{*}\right\} \\
& \left\{a\left(s_{1}^{*}\right)^{\gamma_{2}^{*}-\gamma_{1}^{*}} x^{-\gamma_{2}^{*}}, \quad s_{1}^{*}<x<s_{2}^{*}\right\} \\
& \left\{a\left(s_{1}^{*}\right)^{\gamma_{2}^{*}-\gamma_{1}^{*}} \ldots\left(s_{m}^{*}\right)^{\gamma_{m+1}^{*}-\gamma_{m}^{*}} x^{-\gamma_{m+1}^{*}}, \quad x>s_{m}^{*}\right\}
\end{aligned}
$$

where $a, s_{1}^{*}, \ldots, s_{m}^{*}, R_{1}^{*}\left\{\gamma_{1}^{*}\right\}, \ldots$, and $R_{m+1}^{*}\left\{\gamma_{m+1}^{*}\right\}$ are decided such that $E\left(\varphi_{n}^{*}(x)\right)=1, E\left(S_{t} \varphi_{n}^{*}\left(S_{t}\right)\right) B_{0}=S_{0}$, and $E\left(c_{i}\left(S_{t}\right) \varphi_{n}^{*}\left(S_{t}\right)\right)$ $B_{0}=c_{i 0}, i=1,2, \ldots, n$.

- The lower (upper) bound for options with strike prices between $\left(K_{2 i-1}\right.$, $\left.K_{2 i}\right), i=1,2, \ldots$, is given by the pricing kernel $\varphi_{n}^{* *}\left(S_{t}\right)\left(\varphi_{n}^{*}\left(S_{t}\right)\right)$.

Proof: Let $\bar{R} \rightarrow+\infty$ and $\underline{R} \rightarrow 0 \quad\{\bar{\gamma} \rightarrow+\infty$ and $\underline{\gamma} \rightarrow 0\}$; applying Proposition 3, we immediately obtain the result. Q.E.D.

\section{Conclusions}

In this paper we have derived DARA $\{$ DRRA $\}$ option bounds from concurrently expiring options. We show that these bounds are given by the pricing representative investors who have piecewise CARA $\{\mathrm{CRRA}\}$. 
It has been generally accepted that investors have DARA. Some empirical findings also suggest that the pricing representative investor may have DRRA. Thus the DARA and DRRA option bounds are practically meaningful.

The results have important implications for arbitrage opportunities in the markets of concurrently expiring options. If the option bounds derived in this paper are violated then we can construct an arbitrage portfolio to take the advantage.

Since this paper reveals the relationship between option pricing bounds and the bounds of risk aversion of the pricing representative investor, it also presents a potential method for backing out some characteristics of investors' utility functions. 


\section{REFERENCES}

1. Arrow, Kenneth J. (1971). Essays in the Theory of Risk Bearing. Amsterdam: North Holland.

2. Basso, Antonella and Paolo Pianca (1997). "Decreasing Absolute Risk Aversion and Option Pricing Bounds." Management Science 43, 206-216.

3. Benninga, S. and J. Mayshar (2000). "Heterogeneity and Option Pricing." Review of Derivatives Research 4, pp. 7-27.

4. Bernardo, Antonio E. and Olivier Ledoit. (2000). "Gain, Loss and Asset Pricing." Journal Political Economy 108, 144-172.

5. Bertsimas, Dimitris and Ioana Popescu (2002). "On the Relation between Option and Stock Prices: A Convex Optimization Approach." Operations Research 50, 358-374.

6. Black, Fischer and Myron Scholes (1973). "The Prices of Options and Corporate Liabilities.” Journal of Political Economy 81, 637-654.

7. Boyle, P. and X. S. Lin (1997). "Bounds on Contingent Claims Based on Several Assets." Journal of Financial Economics 46, 383-400.

8. Brennan, Michael J. (1979). "The pricing of Contingent Claims in Discrete Time Models." Journal of Finance 34, 53-68.

9. Cochrane, John H. and Jesus Saa-Requejo (2000). "Beyond Arbitrage: Good-Deal Asset Price Bounds in Incomplete Markets." Journal Political Economy 108, 79-119. 
10. Constantinides, G. M. and T. Zariphopoulou (1999). "Bounds on prices of contingent claims in an intertemporal economy with proportional transaction costs and general preferences." Finance and Stochastics 3, 345-369.

11. Constantinides, G. M. and T. Zariphopoulou (2001). "Bounds on derivative prices in an intertemporal setting with proportional transaction costs and multiple securities." Mathematical Finance 11 (3), 331-346.

12. Franke, G., R. C. Stapleton, and M. G. Subrahmanyam (1999). "When are Options Overpriced: The Black-Scholes Model and Alternative Characterizations of the Pricing Kernel." European Finance Review 3, 79-102.

13. Garman, M. (1976). "An Algebra for Evaluating Hedge Portfolios." Journal of Financial Economics 3, 403-428.

14. Grundy, Bruce D. (1991). "Option Prices and the Underlying Asset's Return Distribution.” Journal of Finance 46, 1045-1070.

15. Huang, James (2004). "Option Pricing Bounds and the Elasticity of the Pricing Kernel." Review of Derivatives Research 7, 25-51.

16. Huang, James (2004a). "Risk Neutral Probabilities and Option Bounds: A Geometric Approach.” Lancaster University Management School working paper.

17. Huang, James (2004b). "Option Bounds and Second Order Arbitrage Opportunities." Lancaster University Management School working paper. 
18. Huang, James (2004c). "Stochastic Dominance Option Bounds and Nth Order Arbitrage Opportunities.” Lancaster University Management School working paper.

19. Huang, James (2004d). "Option Bounds from Concurrently Expiring Options When Relative Risk Aversion is Bounded." Lancaster University Management School working paper.

20. Huang, Chi-Fu and Robert H. Litzenberger Foundations for Financial Economics, New York: North-Holland 1988.

21. Jackwerth, Jens C. and Mark Rubinstein (1996). "Recovering Probability Distributions from Option Prices.” Journal of Finance 51, 1611-1632.

22. Lo, A. (1987). "Semiparametric Upper Bounds for Option Prices and Expected Payoffs." Journal of Financial Economics 19, 373-388.

23. Masson, Jean and Stylianos Perrakis (2000). "Option Bounds and the Pricing of Volatility Smile." Review of Derivative Research 4, 29-53.

24. Levy, Haim (1985). "Upper and lower Bounds of Put and Call Option Value: Stochastic Dominance Approach." Journal of Finance 40, 11971217.

25. Mathur, Kamlesh. and Peter Ritchken (2000). "Minimum Option Prices Under Decreasing Absolute Risk Aversion." Review of Derivative Research $3,135-156$. 
26. Merton, Robert C. (1973). "Theory of Rational Option Pricing." Bell Journal of Economics and Management Science 4, 141-183.

27. Perrakis, Stylianos and Peter J. Ryan (1984). "Option Pricing Bounds in Discrete Time," Journal of Finance 39, 519-525.

28. Pratt, John W. (1964). "Risk Aversion in the Small and in the Large." Econometrica 32, 122-136.

29. Ritchken, Peter (1985). "On Option Pricing Bounds," Journal of Finance 40, 1219-1233.

30. Ritchken, Peter and Shyanjaw Kuo, (1989). "On Stochastic Dominance and Decreasing Absolute Risk Averse Option Pricing Bounds," Management Science 35, 51-59.

31. Rubinstein, Mark (1976). "The Valuation of Uncertain Income Streams and the Pricing of Options." Bell Journal of Economics 7, 407-425.

32. Ryan, Peter (2000). "A Tighter Option Bounds from Multiple Exercise Prices." Review of Derivatives Research 4, 155-87.

33. Ryan, Peter (2003). "Progressive Option Bounds from the Sequence of Concurrently Expiring Options." European Journal of Operations Research 151, 193-223.

34. Sachdeva, Kanwal (1986). "On the Equality of Two Lower Bounds on the Call Price: A Note.” Journal of Financial and Quantitative Analysis 21, 235-237. 
35. Stapleton, Richard C. and Marti G. Subrahmanyam (1990). "Risk Aversion and the Intertemporal Behavior of Asset Prices." Review of Financial Studies 3, 677-693. 